\title{
Transesophageal Monitoring in Anaesthesia: An Update
}

\author{
Mahesh R. Prabhu • Anthony George
}

Published online: 30 July 2014

(c) Springer Science+Business Media New York 2014

\begin{abstract}
Transesophageal echocardiography (TEE) is increasingly used for diagnosis and monitoring in a number of clinical areas for assessment of cardiac structure and function. The use of TEE is well established in cardiac surgery but in non-cardiac surgery, TEE is recommended in preexistent cardiovascular disease causing hemodynamic compromise or in the presence of refractory hemodynamic instability or hypoxemia. Real-time 3-dimensional TEE provides exceptional images and unique views which have been pivotal in the success of minimally invasive surgery and transcatheter interventions. Although TEE is a relatively safe procedure, guidelines for standardized training, techniques and research protocols are recommended by most august bodies. The use of simulation-based learning has aided training by providing structured training opportunities and sufficient training time. TEE is a semi-invasive, portable, safe and readily available imaging modality that is likely to see an upsurge due to innovations in technology, miniaturization and an increase in minimally invasive procedures.
\end{abstract}

Keywords Transesophageal - Trends - Cardiac surgery · Thoracic surgery $\cdot 3$-dimensional TEE · Interventional/ methods Perioperative period

M. R. Prabhu $(\bowtie) \cdot$ A. George

Department of Cardiothoracic Anaesthesia and Intensive Care, Freeman Hospital, Freeman road, High Heaton, Newcastle upon Tyne NE7 7DN, UK

e-mail: mahesh.prabhu@nuth.nhs.uk

A. George

e-mail: anthony.george@nuth.nhs.uk

\section{Introduction}

Transesophageal echocardiography (TEE) is increasingly used both as diagnostic equipment and a monitoring device in a number of clinical areas including the operating theatre, intensive care unit, interventional laboratory and outpatient setting. TEE is used for the assessment of cardiac structure and function in both cardiac and non-cardiac surgery as well as a guiding tool in transcatheter interventions [1]. In cardiac surgery, TEE is employed to confirm the preoperative diagnosis, detect new or unsuspected pathology, and evaluate the results of surgery (Fig. 1). TEE is progressively used in non-cardiac surgery when the cardiovascular pathology or the planned surgery is likely to cause severe hemodynamic, pulmonary or neurological compromise [2].

The therapeutic impact of TEE is difficult to assess but initiation of cardiac drugs, optimal fluid management, changes in the anaesthetic and surgical plan, and additional surgical procedures after a TEE examination have been shown to be beneficial especially in category I indications $[3,4]$.

Significant advances in technology have made TEE accessible and affordable to clinicians thereby enabling either a comprehensive study or even a limited, "goalfocused" examination aimed at addressing specific clinical concerns $[5 \bullet \cdot$. This article aims to highlight recent trends and developments in TEE, and focuses on the everexpanding list of indications for TEE in anaesthesia.

\section{Appropriateness of TEE in Surgery}

Guidelines have been formulated to ensure that both transthoracic and transesophageal echocardiography are 


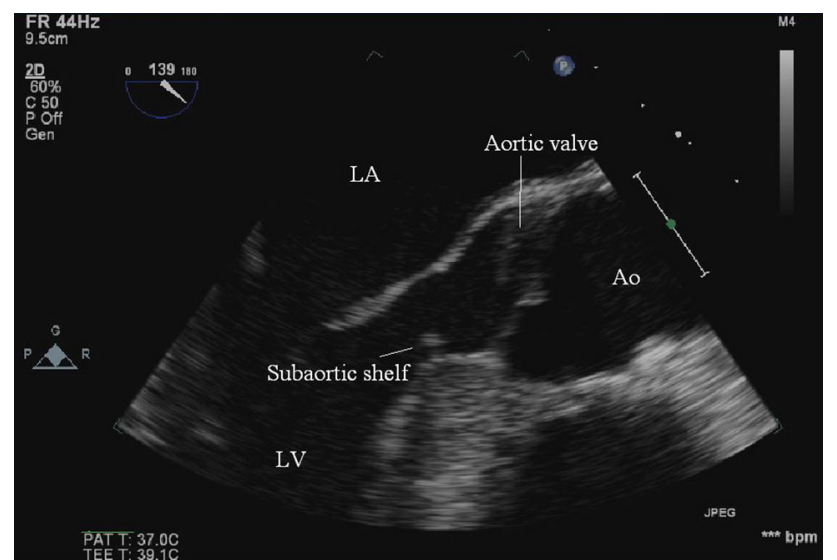

Fig. 1 Incidental finding of sub-aortic shelf seen in the ME aortic valve LAX view causing a degree of previously undiagnosed left ventricular outflow tract obstruction. $L A$ left atrium, $A o$ aorta, $L V$ left ventricle

used appropriately in clinical scenarios [6]. 202 indications have been identified and scored for appropriateness with an aim to improving patient care and health outcomes in a cost-effective manner. Generally, the use of echocardiography is considered to be appropriate when it is used to make a diagnosis, assess a change in clinical status or is likely to influence patient management. This includes the use of TEE to assess valvular abnormalities and to confirm the presence or absence of aortic pathology, intra-cardiac shunts and embolic sources [6].

\section{TEE in Cardiothoracic Surgery}

TEE in cardiac surgery confirms the diagnosis, guides cannulation, assesses surgical results and also assists planning of catheter-based intra-cardiac procedures. TEE is a useful modality for the rapid diagnosis and treatment of thoracic organ injury in blunt and penetrating trauma. The practice guidelines by American Society of Anesthesiologists (ASA) and the Society of Cardiovascular Anesthesiologists (SCA) recommend that, in the absence of contraindications, TEE should be used in all open heart and thoracic aortic surgical procedures and should be considered in certain coronary artery bypass graft surgeries [2] (Fig. 2). In recently published literature, TEE has been shown to be especially useful in the following procedures.

\section{TEE in Aortic Valve Surgery}

Aortic valve repair is a challenging but viable alternative to prosthetic valve replacement in the younger patient with aortic regurgitation (AR). Intraoperative TEE is used to assess aortic valve morphology, severity of AR, mechanisms of AR based on leaflet mobility, as well as foster a systematic approach to surgery [7].

\section{TEE in Thoracic Surgery}

Patients with pre-existing respiratory disease and associated right ventricular (RV) impairment undergoing noncardiac thoracic surgery may benefit from intraoperative TEE monitoring. RV dysfunction and left ventricular diastolic dysfunction (LVDD) have both been implicated as risk factors for atrial arrhythmias following thoracic surgery [8]. TEE has recently been used to assess the effects of intraoperative one-lung ventilation strategies on the right ventricle. RV function appears to be less impaired by pressure-controlled ventilation when compared to volumecontrolled mechanical ventilation [9].

\section{TEE in Thoracic Transplantation Surgery}

TEE is used to evaluate the ventricular function, volume status, surgical anastomoses, pulmonary artery thrombi and concurrent congenital heart defects in thoracic organ transplantation. TEE can also assist in the donor selection process [10]. During single sequential lung transplantation, TEE is used as a hemodynamic monitor to assess the effects of one-lung ventilation and pulmonary artery clamping as well as guide the need for extracorporeal support post transplantation [11•]. Post transplantation, TEE has also been used to diagnose anastomotic stenosis or kinking and pulmonary venous thrombosis [12•] (Fig. 3).

\section{TEE in Extracorporeal Membrane Oxygenation}

Extracorporeal membrane oxygenation (ECMO) provides hemodynamic and/or respiratory support to critically ill patients using a modified cardiopulmonary bypass circuit and large-bore cannulae placed in or near the heart. The two modes used are venovenous (VV) and venoarterial (VA) ECMO. TEE monitoring is used to diagnose anatomical abnormalities of the heart and exclude reversible causes of hemodynamic instability prior to cannulation. It is also used to assist the correct placement of cannulae, monitor the response to ECMO, identify any complications, consider the readiness to wean from ECMO and finally oversee the weaning process [13].

\section{TEE in Pulmonary Hypertension}

Pulmonary hypertension $(\mathrm{PH})$ and RV failure are associated with significant mortality [14]. The major risk factors are preoperative RV impairment, increased pulmonary vascular resistance and the type of surgical procedure [15]. The risk is greatest in surgical procedures associated with 


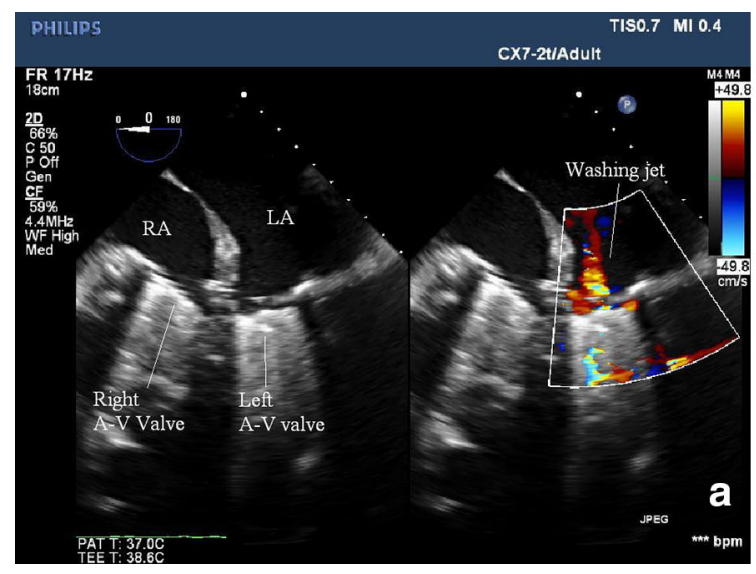

Fig. 2 TEE during implantation of the Syncardia Total Artificial Heart. The equivalent ME 4-chamber view shows the native right and left atria ( $R A$ and $L A)$ and the right and left mechanical
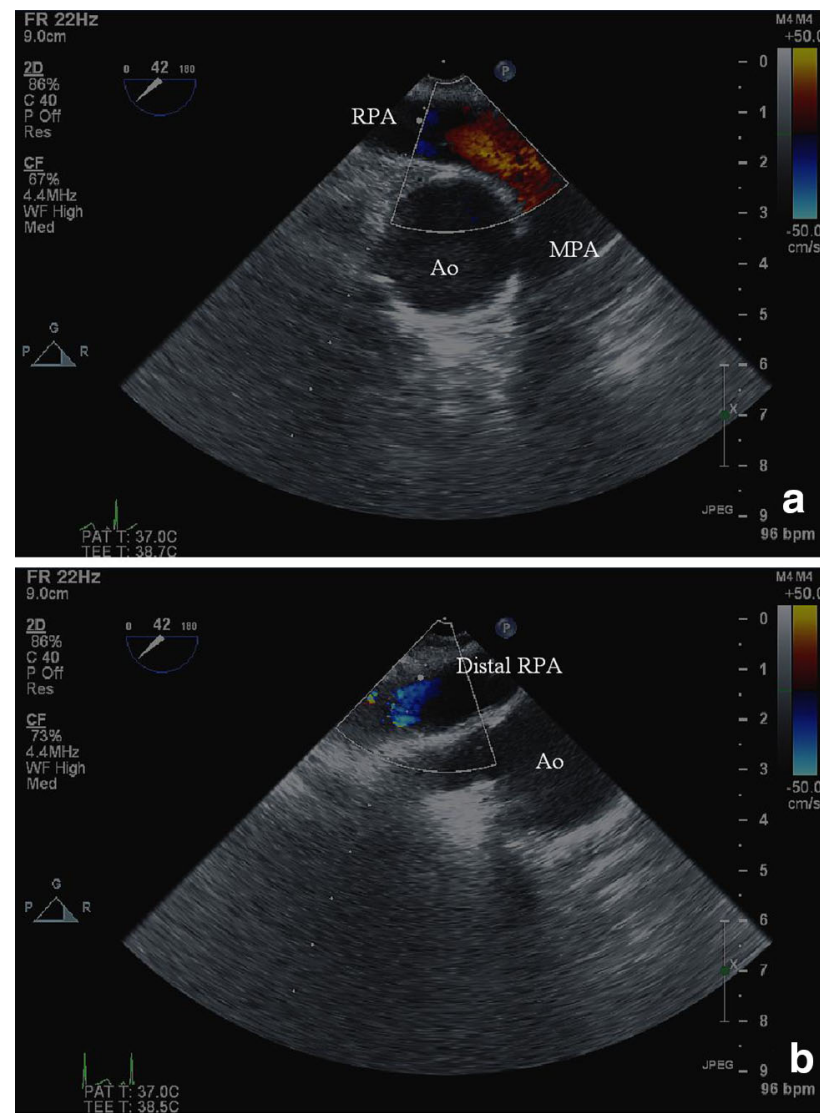

Fig. 3 TEE for lung transplantation surgery. ME ascending aortic SAX view showing the course of the RPA up to the anastomosis. $M P A$ main pulmonary artery, $R P A$ right pulmonary artery, Ao Aorta

marked systemic inflammatory response, rapid blood loss, venous embolism (air, carbon dioxide, fat or cement) and loss of pulmonary vasculature [15].

The 2-dimensional (2D) echocardiographic features and the Doppler indices required for the evaluation of $\mathrm{PH}$ have

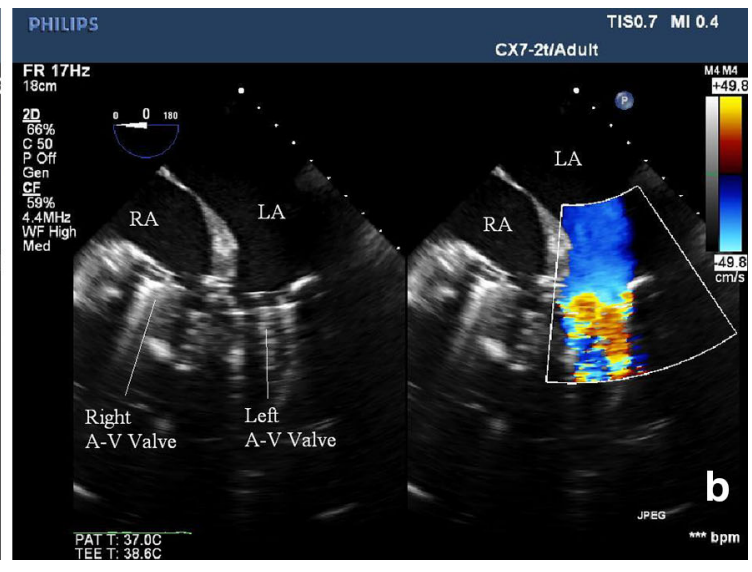

atrioventricular valves (right and left $\mathrm{A}-\mathrm{V}$ valves) in systole and diastole. A central washing jet can be seen with CFD during systole

been extensively reviewed [16]. Chronic RV pressure overload may be diagnosed by enlarged right heart, impaired RV systolic function and a flattened interventricular septum causing a small D-shaped left ventricle [16]. Patients with PH will have significant pulmonary regurgitation with at least moderate tricuspid regurgitation (TR) secondary to tricuspid annular dilatation, altered RV geometry and apical displacement of the tricuspid leaflets [16]. A pulsed-wave Doppler waveform analysis of the pulmonary artery flow velocity profile obtained from one of the upper oesophageal views can provide evidence of increased impedance and/or decreased compliance of the pulmonary vascular tree [17].

\section{TEE in Diastolic Dysfunction}

Changes in loading conditions, arrhythmias, complex measurements and the lack of effective therapy have discouraged echocardiographers from assessing LVDD intraoperatively. $\mathrm{E} / \mathrm{e}^{\prime}$ ratio is an index for LVDD, where $\mathrm{E}$ is early transmitral velocity and $\mathrm{e}^{\prime}$ is the tissue Doppler mitral annular early diastolic velocity (Fig. 4). A simplified TEE algorithm for assessing LVDD using both $\mathrm{e}^{\prime}$ and the $\mathrm{E} / \mathrm{e}^{\prime}$ ratio in CABG patients $(n=905)$ has been predictive of long-term major adverse cardiac events [18]. A practical approach to the assessment of LVDD involving LA size has also been proposed [19]. Simpler algorithms and robust indices are likely to see an increase in diagnosis of LVDD [20••].

\section{TEE in Non-cardiac Surgery}

In the past, use of TEE in non-cardiac surgery has been limited by acquisition costs, lack of training, experience, familiarity and perhaps the lack of comprehension of its true potential [10]. Intraoperative TEE is recommended in 

using $E / e^{\prime}$ ratio. Normal transmitral flow pattern using PWD: peak $E$ velocity, peak $A$ velocity (a). Normal pattern of tissue Doppler imaging of the mitral annulus: peak $e^{\prime}$ velocity, peak $a^{\prime}$ velocity (b)
Fig. 4 Measurement of LVDD
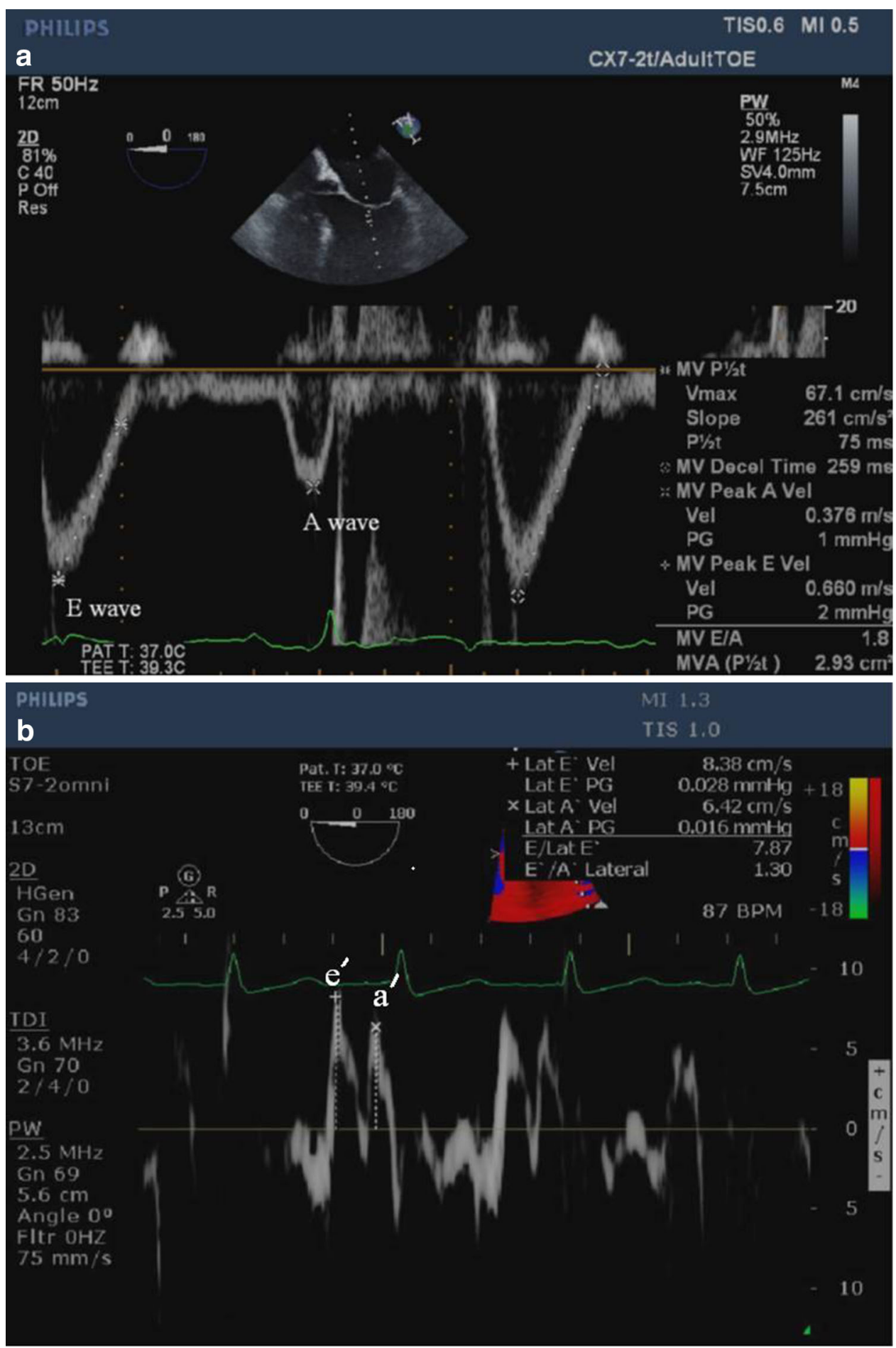

non-cardiac surgery when the patient has known or suspected cardiovascular disease that might result in severe hemodynamic, pulmonary or neurologic compromise or when unexplained and refractory intraoperative hemodynamic instability or hypoxemia occurs. The goal is to determine the cause of hemodynamic instability and to guide therapeutic interventions required to treat the cause [2]. However, there are no outcome studies in which TEE has been randomized as an intervention to assess patient outcome [5••]. Misdiagnosis is also a likely risk and the importance of knowledge, training and experience must be stressed.

\section{TEE in Emergency Surgery}

Rescue echocardiography has been used successfully in patients with severe systemic disease experiencing 
hemodynamic difficulties during non-cardiac surgery. In this single-centre trial $(n=31)$ of patients undergoing predominantly abdominal or orthopaedic surgery, 9 of the 20 standard TEE views were used to assess the cause for the hemodynamic instability [21•]. Rescue echocardiography not only provided a diagnosis but also changed pharmacological and fluid therapy as well as directed emergency secondary procedures such as pericardial drainage, percutaneous coronary intervention and pulmonary thrombectomy. TEE may also have a role in determining the cause of hemodynamic instability in trauma patients unresponsive to fluid resuscitation [22]. TEE helps to diagnose pre-existing cardiac problems and traumatic thoracic injury but the risk of TEE-related viscus rupture potentiated by high gastric volumes or associated traumatic oesophageal injury must be considered.

Recognizing the increasing use of perioperative echocardiography, the recent consensus statement from the American Society of Echocardiography (ASE) and the Society of Cardiovascular Anesthesiologists (SCA) outlines the 11 basic TEE views necessary to perform a noncomprehensive examination in order to diagnose the cause of hemodynamic instability and guide therapeutic interventions [23*0]. The appropriate views required for assessment of ventricular function, hypovolemia, basic valvular lesions, pulmonary embolism, venous air embolism, pericardial effusion, thoracic trauma and simple congenital heart disease in adults are well described.

\section{Vascular Surgery}

TEE is used to diagnose hypovolemia and ischemic myocardium following vascular surgery. Both left ventricular systolic and diastolic dysfunction were significant predictors of postoperative cardiovascular events [24]. TEE provides a fast, precise and portable diagnostic tool in emergency vascular surgery for aortic dissection and disruption. In aortic dissection needing endovascular aortic repair, TEE has been used to identify the true lumen, the landing zone, guide the deployment of the stent and diagnose endoleaks [25].

\section{TEE in Liver Transplantation Surgery}

TEE in high-risk non-cardiac surgery such as liver transplantation is increasingly performed in high-volume transplant centres [26, 27]. A limited upper oesophageal TEE examination is useful in managing fluid therapy, monitoring myocardial function and identifying intraoperative complications, including heart failure, even in the setting of distal oesophageal pathology [28]. Intraoperatively, the effects of major phases of the surgical procedure (dissection phase, venovenous bypass, anhepatic phase and reperfusion phase) can be monitored by TEE [29].
Obstetrics

Cardiac disease is the leading cause of maternal mortality in developing countries. The use of TEE as a monitoring tool in acute, severe hemodynamic instability in the peripartum period has been limited to case reports [30]. TEE has been used in high-risk obstetric patients with pulmonary hypertension or congenital heart disease or in the diagnosis of massive amniotic fluid embolism [31].

\section{3-Dimensional Transesophageal Echocardiography}

Real-time (RT) 3-dimensional (3D) TEE is a major advance that has revolutionized our understanding of complex anatomical structures. It provides exceptional images promoting better understanding of the relationship between cardiac structures, as well as accurate quantification of cardiac function (Fig. 5).

2D TEE has been the backbone of echocardiographic imaging but one of the main limitations has been the inability to visualize the entire structure. For example, various methodologies to image the mitral valve do not take into account the physiological and pathological variations in anatomy. In fact, haptic knowledge of the TEE probe adjustments needed to acquire an image was found to be crucial in identifying the mitral scallops accurately [32].

Although 3D technology has been around for over a couple of decades, recent advances in technology such as multilinear probes, volumetric dataset acquisition and offline analysis have enhanced our understanding of the heart [33]. 3D TEE has made it easier to interrogate, interpret and understand complex structures such as the mitral valve and right ventricle. This in-depth knowledge of pathophysiological anatomy is crucial for success in surgical techniques especially the minimally invasive and transcatheter approaches. Live 3D TEE has helped to direct these interventions by intuitively guiding these procedures [34]. Use of augmented reality in ultrasound to guide interventions is likely to improve this experience in the future [35].

\section{D TEE of the Mitral Valve}

The mitral annulus (MA) is a nonplanar, saddle-shaped, hyperbolic paraboloid fibrous ring [36*0]. Comprehensive 3D analysis of the mitral valve (MV) using customized software has been used to generate high-resolution, consistent 3D models with very little inter-operator and intraoperator variability [37]. Evaluation of the complex morphology and dynamics of the MV using different types of computational, geometrical and biomechanical models is likely to lead to a better understanding of the MV [38]. In 

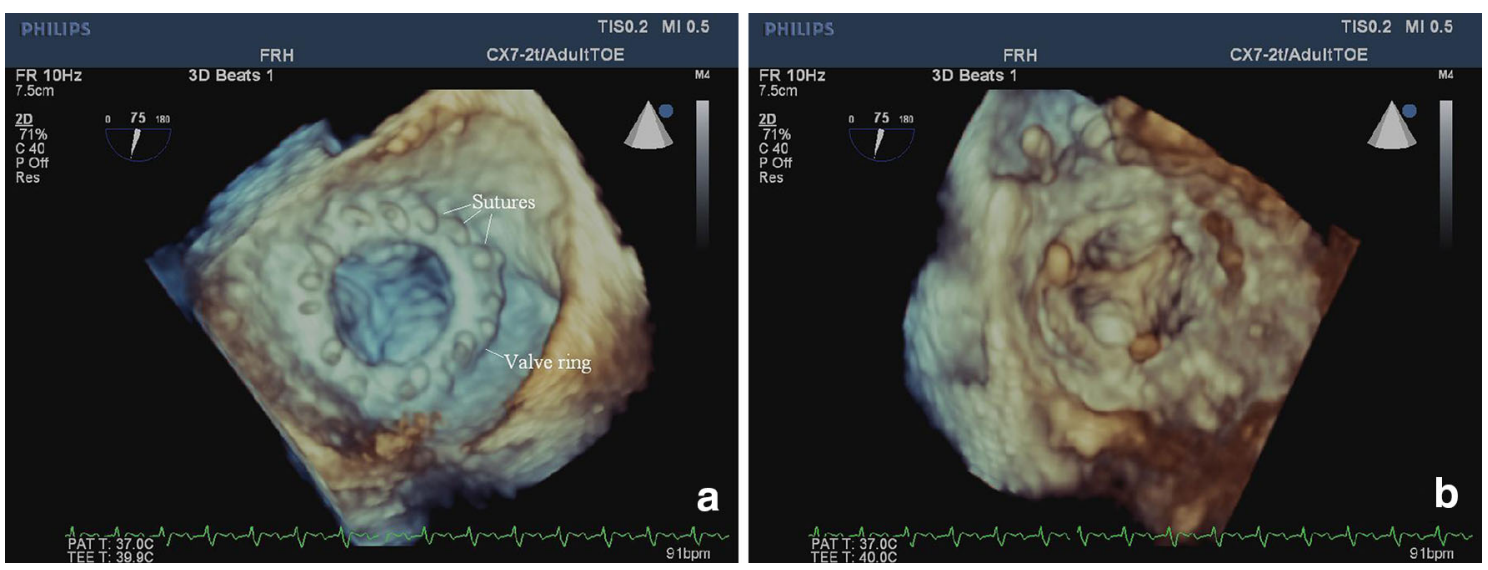

Fig. 5 RT 3D TEE of a bioprosthetic mitral valve replacement as visualized from the left atrium (a) and left ventricle (b). In a, the sutures and the valve ring of the bioprosthetic valve are clearly visible

mitral or ventricular pathology, the MA may be dilated, deformed or flattened causing a loss of the saddle shape leading to inefficient contraction during systole and valvular incompetence $[39,40]$.

\section{D TEE of the Right Heart}

Right heart dysfunction in the perioperative period is associated with poor outcomes. Numerous publications have described the views on the echocardiographic evaluation of the right heart [41-43]. 3D TEE has helped in understanding the irregular, complex anatomy of the right heart by visualizing the entire RV and eradicating geometric assumptions. 3D TEE datasets on patients undergoing cardiac surgery offer an emerging and validated approach to assess RV stroke volume [44•]. Customized software creates a surface-rendering cast of the RV and helps measure end-diastolic and end-systolic volumes as well as segmental analyses of the inlet, apex and outflow segments. Measuring the tricuspid annular area using 3D planimetry has been shown to be more accurate than the conventional linear ellipse method [45].

\section{D TEE in Congenital Heart Disease}

Congential heart disease (CHD) is recognized for its complex intra-cardiac anatomy and intricate 3-dimensional correlations [46]. Careful acquisition, expert evaluation and interpretation of multiple images are needed when using 2D imaging [47]. 3D imaging not only simplifies this process but also provides additional value and improved image quality $[48,49]$. The en face views using 3D technology provide a unique perspective of the complex morphology and pathophysiology of congenital defects [50]. Multiplanar reconstruction (MPR) provides access to an unlimited number of 2D echocardiographic planes. 3D echocardiographic measurement of ventricular volumetrics has been shown to have good correlation with Magnetic resonance imaging [51, 52]. High-quality $3 \mathrm{D}$ TEE is increasingly being used for diagnosis, assessment and guidance of transcatheter interventions with good effect.

\section{TEE for Transcatheter Interventions (TCI)}

Echocardiography in patients undergoing TCI enhances decision making, shortens fluoroscopy time and reduces procedural complication rates (Fig. 6). Backed by observational studies, the ASA/SCA practice guidelines recommend that TEE may be used in patients undergoing transcatheter interventions [2]. The European Association of Echocardiography (EAE) and the ASE have also published recommendations for the use of echocardiography in TCI for valvular heart disease [53••]. 3D TEE will continue to play an indispensible role in this rapidly emerging discipline of transcatheter interventions but the limitations include a significant learning curve, cost and limited temporal resolution.

\section{Transcatheter Aortic Valve Implantation}

TEE is useful in patient selection, choice of procedure and prosthesis, monitoring during the procedure as well as detection of complications. TEE helps evaluate LVOT and aortic root anatomy, aortic annular size, valve opening, the number, mobility and thickness of cusps, extent and distribution of calcification, distance from the aortic annulus to coronary ostia and aortic atheroma [53••]. There is no consensus regarding the gold standard imaging technique for annular sizing but TEE measurements correlate well with TTE [54]. The depth perspective of 3D TEE has aided us to understand the anatomy of the annulus and to visualize the deployment of the prosthesis. 

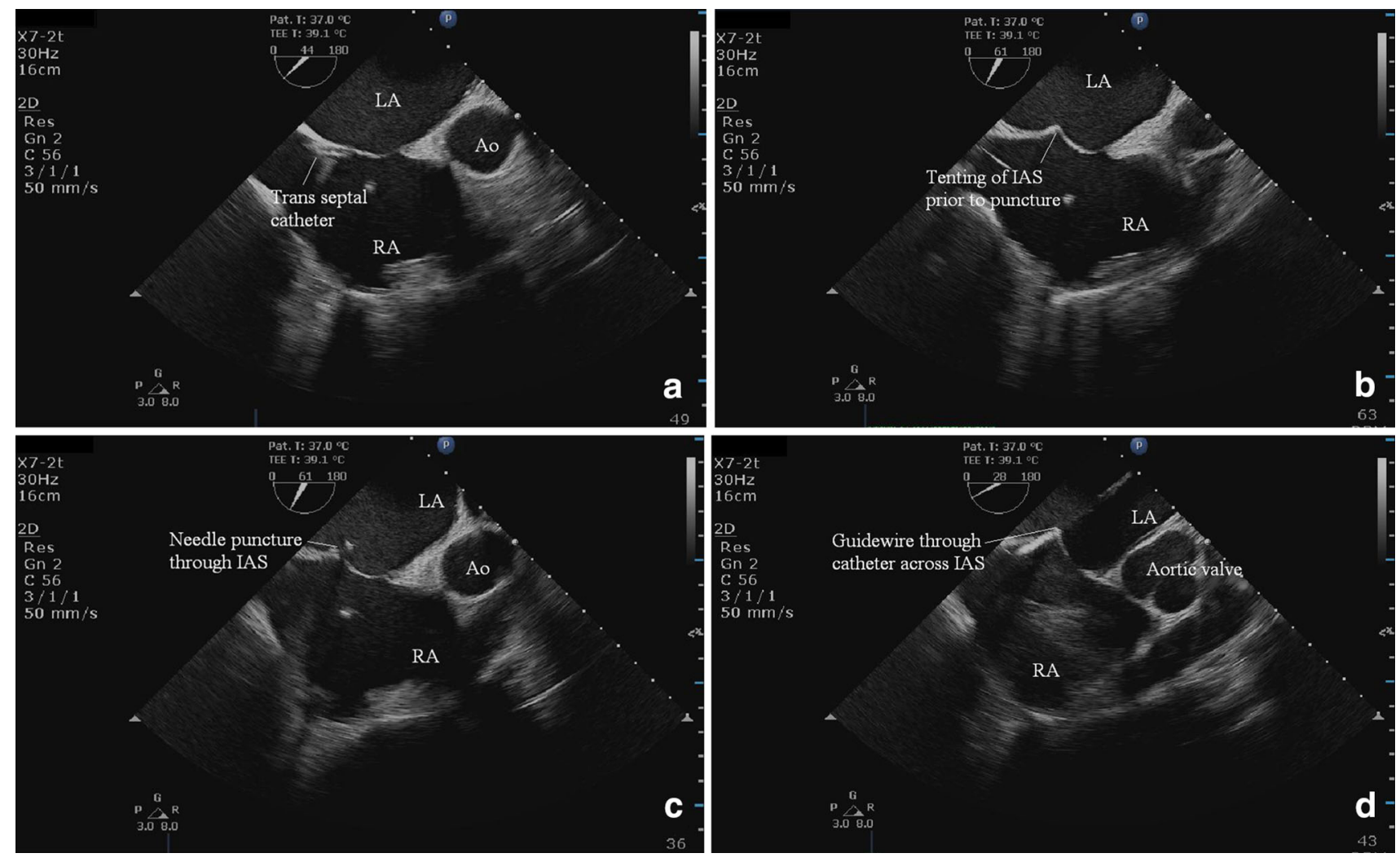

Fig. 6 TEE-guided trans-septal puncture for catheter-based cardiac procedures. In this mid-esophageal view of the inter-atrial septum (IAS), the catheter can be seen in the right atrium (a). The needle

causes tenting of the IAS before puncturing it $(\mathbf{b}, \mathbf{c})$. The guidewire can be seen across the IAS (d). $R A$ right atrium, $L A$ left atrium, $A o$ Aorta

TEE is also used to position the balloon and prosthesis, assess prosthetic function and detect complications such as prosthesis misplacement and paravalvular regurgitation (Fig. 7). Improvements in transnasal TEE and intra-cardiac echocardiography technology may obviate the need for general anaesthesia in the future [55].

\section{Percutaneous Mitral Valve Intervention}

Percutaneous mitral valve intervention is used to repair the mitral valve in high-risk patients. Echocardiography is necessary to assess the functional anatomy, the mechanism of mitral regurgitation (MR) and guide the procedure. The different techniques include indirect and direct annuloplasty, leaflet repair and ventricular remodelling [53••]. In the percutaneous edge-to-edge repair using Mitraclip, a coapting surface length $>2 \mathrm{~mm}$, coaptation depth of $<11 \mathrm{~mm}$, flail height of $\leq 10 \mathrm{~mm}$ and flail width of $\leq 15 \mathrm{~mm}$ are necessary to achieve optimum results [56]. 3D TEE is invaluable during all stages of the procedure including trans-septal catheterization, positioning and orienting the clip above the regurgitant orifice and grasping of the edges to achieve a double-orifice mitral valve.

Paravalvular Regurgitation (PVR)

Real-time 3D-TEE is the preferred mode to guide closure of paravalvular leaks [57]. A recent increase in the number of devices and procedures belies the fact that success may be restrained by technical challenges or the arbitrary nature of the defects. 3D-TEE analysis can be used to generate multiple views of valve dehiscence (Fig. 8). Using the zoom mode and full-volume wide-angle acquisition with colour flow Doppler, the vena contracta of the leaking jet can be analysed for defect location, size and selection of closure device. 3D-TEE can also generate en face views to direct the guidewire and the device, confirm stability and identify complications such as air embolism or tamponade.

\section{Improvements in Technology}

\section{Miniaturization}

A miniaturized, single-use, hemodynamic TEE probe (ImaCor, Garden City, NY, USA) has been used as a pointof-care device to aid placement of cannulae, assessment of 

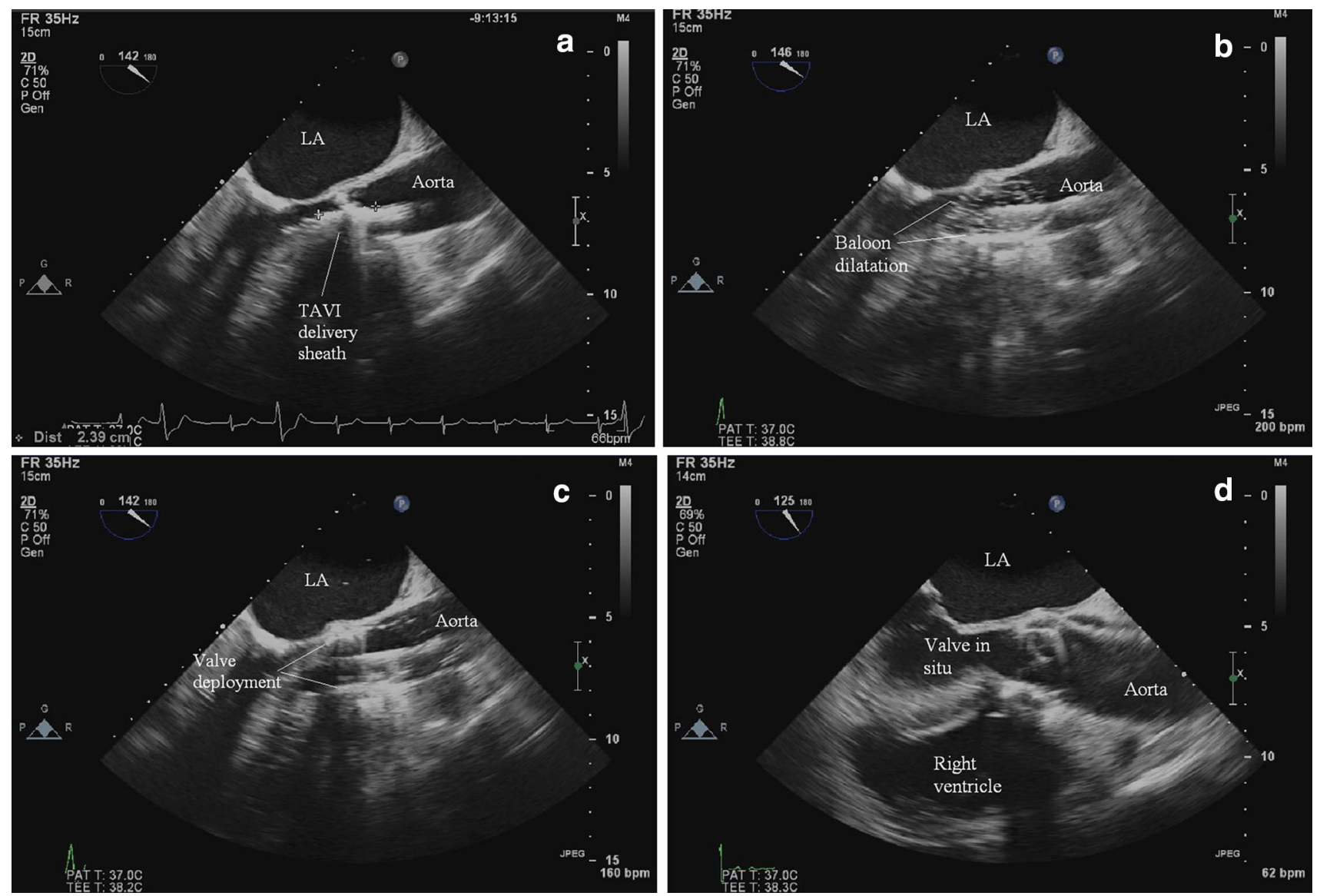

d

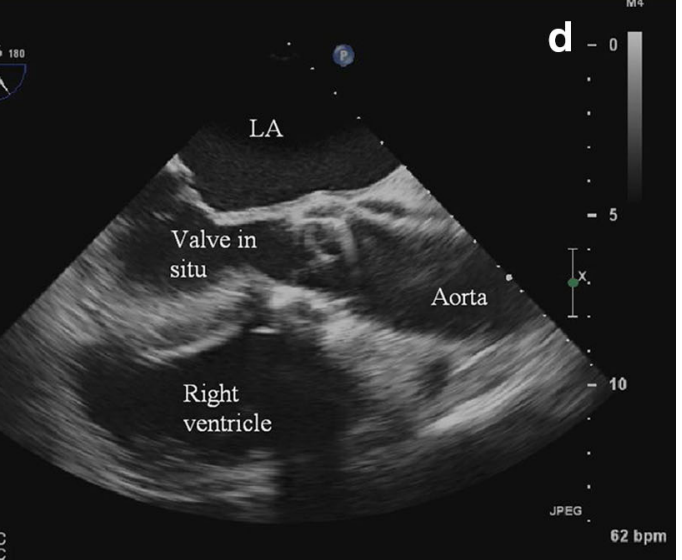

Fig. 7 Transcatheter aortic valve implantation. In this ME aortic valve LAX view, the delivery sheath can be seen across the aortic valve (a). After balloon dilatation (b), the valve is deployed (c). The newly implanted valve can be seen in position (d). $L A$ left atrium
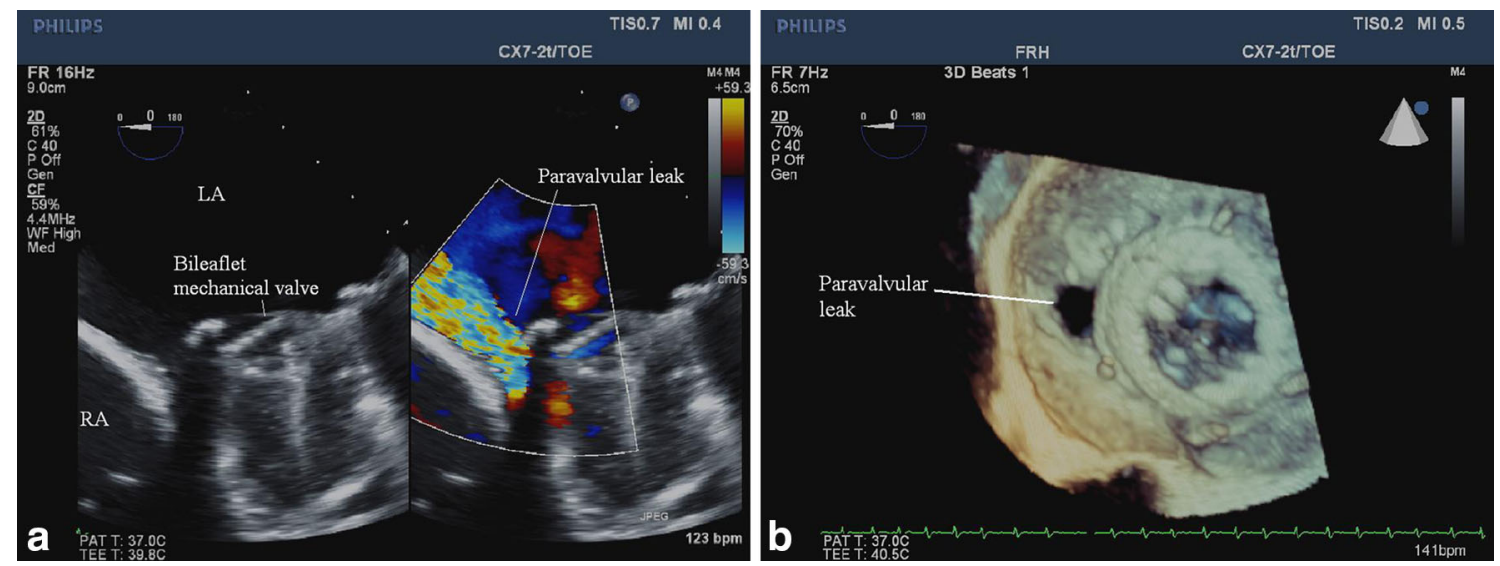

Fig. 8 Paravalvular regurgitation. ME 4-chamber view of the dehisced mechanical mitral valve replacement showing severe PVR using CFD (a). RT 3D TEE of the dehisced mitral valve replacement as visualized from the left atrium (b)

volume status, hemodynamics and valvular pathophysiology. The probe can be left in the oesophagus for up to $72 \mathrm{~h}$ for a focused, intermittent, hemodynamic assessment. It has been used in hemodynamically unstable postoperative cardiac surgical patients, liver transplantation and weaning from ECMO [58, 59].

\section{Myocardial Strain}

Assessment of ventricular function using the traditional volumetric and linear measures of left ventricular systolic function has limitations, and techniques to quantify myocardial deformation have been developed [60]. 
Speckle-tracking echocardiography (STE) is a more objective method which analyses myocardial motion by tracking blocks of 20-40 pixels containing stable echo patterns (speckles) within an ultrasonic window in consecutive frames. An image-processing algorithm tracks these speckles and provides angle-independent sequences of tissue motion and deformation [61]. Several parameters including strain, strain rate, torsional deformation and twist can be measured [62].

A recent review of the use of STE during cardiac surgery looked at the ability of STE to assess ventricular function intraoperatively [60]. Strain analysis has minimized inter- and intraobserver variability in detection of contractile dysfunction compared to traditional wall motion scoring systems [63]. Strain measurements obtained using TEE also correlate well with TTE measurements; however, no reference values exist for equivalence [64, 65].

\section{Education and Training}

\section{Guidelines}

There have been several published guidelines in recent years by various organizations on training, performance and appropriateness of TEE $[1,6,53 \cdot \bullet]$. These guidelines are likely to standardize training, techniques, image acquisition and research protocols. Recent ASE guidelines for performing a comprehensive TEE examination require 28 imaging views with a suggested protocol of image acquisition [66*0. They also underline the importance of knowledge, formal guidelines and training pathways to achieve competency in basic TEE.

\section{Simulation-Based Medical Education}

Training in image acquisition needs skills to deal with a complex interface among the echocardiographer, the machine and the patient. In-depth knowledge of cardiac anatomy, physiology, physics of ultrasound and repetitive, hands-on experience is essential to gain proficiency in image acquisition. Training in echocardiography is often limited by lack of structured training opportunities and sufficient regular training time [67•]. Simulation-based medical education has been shown to minimize patient risks, discomfort and potential distraction in high-stress clinical environments [68, 69]. The echocardiographic simulators available include online software programmes or mannequin-based echocardiographic (TEE \& TTE) simulators $\left[70^{\bullet}\right]$.

The online software programmes are usually free webbased simulation programmes that help in image orientation [71]. The virtual TEE probe is manipulated by either mouse or keyboard controls. The scan plane of the probe slices through the virtual heart model or computerized tomographic datasets and the TEE views are seen on the interface. The main disadvantage of these online software programmes is the lack of actual tactile sensation that comes with the handling of a TEE/TTE probe.

The mannequin-based simulators contain a life-sized mannequin with a model heart or a virtual heart placed within an electromagnetic field. The motion sensor inside the echocardiographic probe tip determines the position and movement of the probe within that electromagnetic field. This position of the probe tip is then used by simulation software to generate 2D echocardiographic images from the virtual heart [72]. The software controls allow for manipulation of the images similar to the TEE machine. Normal anatomical and pathological software modules are available [73]. Moreover, the haptic experience gained by handling and manipulation of the probe is a major advantage of these simulators. One of the main disadvantages is the excessive cost of echocardiographic simulators.

\section{Metrics}

Image acquisition in echocardiography require small, multiple, instinctive probe adjustments which need considerable manual dexterity. Metrics are defined as a set of tools to track and objectively quantify repeat performances of a predefined action [74]. A unique echocardiographic metrics system, Vimedix simulation system (CAE Healthcare Inc., Montreal, Canada) can now measure the time taken to acquire an image (time metrics), track the probe movements made in $3 \mathrm{D}$ space and compare the acquired image to the ideal image (motion metrics). Also, the recorded image can be compared in quality with other operators and experts (observer metrics). Kinematic analysis of probe motion has been used to track the motion of the probe in the $x, y$ and $z$ axes in relation to a virtual heart model [75]. Metrics can be used for performance evaluation and research purposes, and is likely to transform simulation-based training in echocardiography. [76]

\section{Safety of TEE}

Although TEE procedures have been shown to be relatively safe with a low incidence of serious gastroesophageal injury (0.03-0.09\%), TEE-related complications are commonly associated with medicolegal litigation [77]. This is partly due to the devastating morbidity and mortality $(10-56 \%)$ caused by complications such as oesophageal perforation and partly due to the delay in diagnosis and treatment of this insidious complication [78, 79]. Gastroesophageal pathology, distorted anatomy and resistance to 
probe insertion are major risk factors [80]. More importantly, oesophageal perforation can even occur in patients without major risk factors or after an uncomplicated TEE procedure. The risk factors in these patients are elderly age, female sex, small stature and TEE in the operative setting [77]. Listing oesophageal perforation as a specific risk in the informed consent may limit medicolegal liability [79]. Major bleeding complications after TEE with an incidence of $0.02-1.0 \%$ may occur secondary to direct trauma or disruption of friable tissues (e.g. oesophageal varices, oesophageal tumours) [78]. Oesophageal varices, however, are not an absolute contraindication to TEE if correct precautions are taken [29]. Rigid laryngoscopy can reduce the incidence of odynophagia and oropharyngeal mucosal laceration in patients when blind insertion of the TEE probe has proved difficult [81].

\section{Future Directions}

Advances in the future are likely to include improvements in miniaturization, closed-loop systems, customized quantification software, augmented reality, integrated multimodality imaging and telediagnosis. Ageing populations, increased incidence of obesity, type- 2 diabetes and cardiovascular diseases are likely to increase the use of echocardiography. High-quality TEE in minimally invasive surgery, transcatheter interventions and CHD will be in great demand as these novel indications will continue to pose new challenges.

\section{Miniaturization}

Integrated, small, robust, inexpensive, high-quality imaging consoles and miniaturized probes with the capacity to provide excellent images are likely to improve bed-side, clinical decision making. Refinements in transducer technology will make high-resolution 3D TEE available across the spectrum of patient sizes [46].

\section{Technological Innovations}

The exponential growth in technological innovation together with an ever-improving understanding of human physiology is likely to herald significant changes in the future. Innovations in medical technology, microprocessing power, informatics and systems automation will no doubt boost these advances. For example, using battery or solar power as an energy source for ultrasound has seen an upsurge in the identification of heart disease in a low-infrastructure, developing world environment [82].
Training

Standardization of technique, quantification and even equipment according to guidelines set by august bodies will minimize errors, underperformance and aid training of novices. Quantitative assessments will be used to reduce variance in interpretation.

\section{Conclusions}

TEE is a semi-invasive, portable, safe and readily available imaging modality which provides real-time, unique clinical information in the intraoperative setting. There has been an exponential growth in the use of TEE in surgical patients. However, there are no studies in which TEE has been randomized as an intervention to assess patient outcome. The lack of good quality evidence has not dampened the enthusiasm with which the modality has been embraced by the medical community. Indeed, given its wide-spread use, it would probably be unethical and impractical to conduct a blinded randomized controlled trial. A systematic approach to image acquisition and interpretation, improvements in technology, miniaturization and an increase in minimally invasive procedures is likely to see the spread of perioperative TEE in the future.

\section{Compliance with Ethics Guidelines}

Conflict of Interest Mahesh R. Prabhu and Anthony George declare that they have no conflict of interest.

Human and Animal Rights and Informed Consent This article does not contain any studies with human or animal subjects performed by any of the authors.

\section{References}

Papers of particular interest, published recently, have been highlighted as:

- Of importance

•• Of major importance

1. Flachskampf FA, Badano L, Daniel WG, et al. European Association of Echocardiography; Echo Committee of the European Association of Cardiothoracic Anaesthesiologists. Recommendations for transoesophageal echocardiography: update 2010. Eur J Echocardiogr 2010;11:557-76

2. Thys DM, Brooker RF, Cahalan MK, et al. Practice guidelines for perioperative transesophageal echocardiography. An updated report by the American Society of Anesthesiologists and the Society of Cardiovascular Anesthesiologists Task Force on Transesophageal Echocardiography. Anesthesiology. 2010;112: 1084-96. 
3. Denault A, Couture P, McKenty S, et al. Perioperative use of transesophageal echocardiography by anesthesiologists: impact in noncardiac surgery and in the intensive care unit. Can J Anesth. 2002;49:287-93.

4. Hofer C, Zollinger A, Rak M, et al. Therapeutic impact of intraoperative transoesophageal echocardiography during noncardiac surgery. Anaesthesia. 2004;59:3-9.

5. - Royse CF, Canty DJ, Faris J et al. core review: physicianperformed ultrasound: the time has come for routine use in acute care medicine. Anesth Analg 2012;115:1007-28. A comprehensive review of the role of point-of-care ultrasound in perioperative medicine.

6. Douglas PS, Garcia MJ, Haines DE, et al. ACCF/ASE/AHA/ ASNC/HFSA/HRS/SCAI/SCCM/SCCT/SCMR 2011 Appropriate Use Criteria for Echocardiography. A Report of the American College of Cardiology Foundation Appropriate Use Criteria Task Force, American Society of Echocardiography, American Heart Association, American Society of Nuclear Cardiology, Heart Failure Society of America, Heart Rhythm Society, Society for Cardiovascular Angiography and Interventions, Society of Critical Care Medicine, Society of Cardiovascular Computed Tomography, Society for Cardiovascular Magnetic Resonance American College of Chest Physicians. J Am Soc Echocardiogr 2011;24:229-67

7. Vanoverschelde JL, van Dyck M, Gerber B, et al. The role of echocardiography in aortic valve repair. Ann Cardiothorac Surg. 2013;2:65-72.

8. Matyal R, Mahmood F, Hess P, et al. Right ventricular echocardiographic predictors of postoperative supraventricular arrhythmias after thoracic surgery: a pilot study. Ann Thorac Surg. 2010;90:1080-7.

9. Al Shehri AM, El-Tahan MR, Al Metwally R, Qutub H et al. Right ventricular function during one-lung ventilation: effects of pressure-controlled and volume-controlled ventilation. J Cardiothorac Vasc Anesth 2014. doi:10.1053/j.jvca.2013.09.012.

10. Mahmood F, Christie A, Matyal R. Transesophageal echocardiography and noncardiac surgery. Semin Cardiothorac Vasc Anesth. 2008;12:265-89.

11. - Sullivan B, Puskas F, Fernandes-Bustamante A. Transesophageal echocardiography in noncardiac thoracic surgery. Anesthesiol Clin 2012;30:657-69. A review on the use of TEE in high-risk thoracic surgery.

12. - Cartwright BL, Jackson A, Cooper J. Intraoperative pulmonary vein examination by transesophageal echocardiography: an anatomic update and review of utility. J Cardiothorac Vasc Anesth 2013;27:111-20. A comprehensive description on how to obtain traditionally difficult, but important, views of pulmonary veins.

13. Platts DG, Sedgwick JF, Burstow DJ, et al. The role of echocardiography in the management of patients supported by extracorporeal membrane oxygenation. J Am Soc Echocardiogr. 2012;25:131-41.

14. Denault AY, Tardif JC, Mazer CD, Lambert J, for the BART Investigators. Difficult and complex separation from cardiopulmonary bypass in high-risk cardiac surgical patients: a multicenter study. J Cardiothorac Vasc Anesth 2012;26:608-16.

15. Strumpher J, Jacobsohn E. Pulmonary hypertension and right ventricular dysfunction: physiology and perioperative management. J Cardiothorac Vasc Anesth. 2011;25:687-704.

16. Bossone E, D'Andrea A, D'Alto M, et al. Echocardiography in pulmonary arterial hypertension: from diagnosis to prognosis. J Am Soc Echocardiogr. 2013;26:1-14.

17. Hu R, Tousignant C, Chen R. Flow velocity patterns in the pulmonary artery and pulmonary hypertension. Can J Anesth. 2012; 59:716-9.

18. Swaminathan M, Nicoara A, Phillips-Bute BG, et al. Utility of a simple algorithm to grade diastolic dysfunction and predict outcome after coronary artery bypass graft surgery. Ann Thorac Surg. 2011;91:1844-50.

19. Mahmood F, Jainandunsing J, Matyal R. A practical approach to echocardiographic assessment of perioperative diastolic dysfunction. J Cardiothorac Vasc Anesth. 2012;26:1115-23.

20. •• Nicoara A, Whitener G, Swaminathan M. Perioperative diastolic dysfunction: a comprehensive approach to assessment by transesophageal echocardiography. Semin Cardiothorac Vasc Anesth. 2013;18:218-36. An excellent review on applying echocardiographic findings of diastolic dysfunction intraoperatively.

21. - Shillcutt S, Markin N, Montzingo C et al. Use of rapid rescue perioperative echocardiography to improve outcomes after hemodynamic instability in non-cardiac surgical patients. J Cardiothorac Vasc Anesth 2012;26:362-70. In this prospective study, the authors demonstrate the usefulness of intraoperative TEE to guide clinical decision-making in hemodynamically unstable patients.

22. Rebel A, Klimkina O, Hassan ZU. Transesophageal echocardiography for the noncardiac surgical patient. Int Surg. 2012;97: $43-55$.

23. - Reeves S, Finley A, Skubas $\mathrm{N}$ et al. Basic perioperative transesophageal echocardiography examination: a consensus statement of the American Society of Echocardiography and the Society of Cardiovascular Anesthesiologists. Anesth Analg 2013;117:543-58. New guidelines on accreditation for a basic intraoperative TEE examination.

24. Flu WJ, von Kujik JP, Hoeks SE, et al. Prognostic implications of asymptomatic left ventricular dysfunction in patients undergoing vascular surgery. Anesthesiology. 2010;112:1316-24.

25. Grabenwöger M, Alfonso F, Bachet J, et al. Thoracic Endovascular Aortic Repair (TEVAR) for the treatment of aortic diseases: a position statement from the European Association for CardioThoracic Surgery (EACTS) and the European Society of Cardiology (ESC), in collaboration with the European Association of Percutaneous Cardiovascular Interventions (EAPCI). Eur Heart J. 2012;33:1558-63.

26. Soong W, Sherwani SS, Ault ML, et al. United States practice patterns in the use of transesophageal echocardiography during adult liver transplantation. J Cardiothorac Vasc Anesth 2014. doi:10.1053/j.jvca.2013.10.011.

27. Valentine E, Gregorits M, Gutsche JT, et al. Clinical update in liver transplantation. J Cardiothorac Vasc Anesth. 2013;27: 809-15.

28. Spier BJ, Larue SJ, Teelin TC, et al. Review of complications in a series of patients with known gastro-esophageal varices undergoing transesophageal echocardiography. J Am Soc Echocardiogr. 2009;22:396-400.

29. Robertson A, Eagle S. Transesophageal echocardiography during orthotopic liver transplantation: maximizing information without the distraction. J Cardiothorac Vasc Anesth. 2014;28:141-54.

30. Ecker JL, Solt K, Fitzsimons MG, et al. Case 40-2012-a 43-year-old woman with cardiorespiratory arrest after a cesarean section. N Engl J Med. 2012;367:2528-36.

31. James CF, Feinglass NG, Menke DM, et al. Massive amniotic fluid embolism: diagnosis aided by emergency transesophageal echocardiography. Int J Obstet Anesth. 2004;13:279-83.

32. - Mahmood F, Hess PE, Matyal R et al. Echocardiographic anatomy of the mitral valve: a critical appraisal of 2-dimensional imaging protocols with a 3-dimensional perspective. J Cardiothorac Vasc Anesth. 2012;26:777-84. This interesting study highlights the limitations of traditional $2 D$ echocardiographic mitral valve examination methodologies, which do not account for patient specific TEE probe adjustments made during the TEE examination.

33. Urheim S, Andersen K, Aakhus S. Three-dimensional ultrasound in cardiological diagnostics. Tidsskr Nor Laegeforen. 2012;132: 2171-4. 
34. Balzer J, van Hall S, Rassaf T, et al. Feasibility, safety, and efficacy of real-time three-dimensional transoesophageal echocardiography for guiding device closure of interatrial communications: initial clinical experience and impact on radiation exposure. Eur J Echocardiogr. 2010;11:1-8.

35. Bainbridge D. The use of ultrasound to guide interventions: from bench to bedside and back again. Semin Cardiothorac Vasc Anesth. 2010;14:183-6.

36. • Mahmood F, Shakil O, Mahmood B et al. Mitral annulus: an intraoperative echocardiographic perspective. J Cardiothorac Vasc Anesth 2013;27:1355-63. A comprehensive description of mitral annular anatomy and how to assess normal and abnormal anatomy using TEE.

37. Jassar AS, Brinster CJ, Vergnat M, et al. Quantitative mitral valve modeling using real-time three-dimensional echocardiography: technique and repeatability. Ann Thorac Surg. 2011;91:165-71.

38. Noack T, Kiefer P, Ionasec R, et al. New concepts for mitral valve imaging. Ann Cardiothorac Surg. 2013;2:787-95.

39. Silbiger JJ. Mechanistic insights into ischemic mitral regurgitation: echocardiographic and surgical implications. J Am Soc Echocardiogr. 2011;24:707-19.

40. Khabbaz KR, Mahmood F, Shakil O, et al. Dynamic 3-dimensional echocardiographic assessment of mitral annular geometry in patients with functional mitral regurgitation. Ann Thorac Surg. 2013;95:105-10.

41. Haddad F, Couture P, Tousignant C, et al. The right ventricle in cardiac surgery, a perioperative perspective. I. Anatomy, physiology, and assessment. Anesth Analg. 2009;108:407-21.

42. Rudski LG, Lai WW, Afilalo J, et al. Guidelines for the echocardiographic assessment of the right heart in adults: a report from the American Society of Echocardiography endorsed by the European Association of Echocardiography, a registered branch of the European Society of Cardiology, and the Canadian Society of Echocardiography. J Am Soc Echocardiogr. 2010;23:685-713.

43. Kasper J, Bolliger D, Skarvan K, et al. Additional cross-sectional transesophageal echocardiography views improve perioperative right heart assessment. Anesthesiology. 2012;117:726-34.

44. - Karhausen J, Dudaryk R, Phillips-Bute B et al. Three-dimensional transesophageal echocardiography for perioperative right ventricular assessment. Ann Thorac Surg 2012;94:468-74. Emerging three-dimensional imaging techniques are applied to intraoperative assessment of right ventricular function.

45. Mahmood F, Kim H, Chaudary B, et al. Tricuspid annular geometry: a three-dimensional transesophageal echocardiographic study. J Cardiothorac Vasc Anesth. 2013;27:639-46.

46. Shirali G. Three-dimensional echocardiography in congenital heart disease. Echocardiography. 2012;29:242-7.

47. Ayres NA, Miller-Hance W, Fyfe DA, et al. Pediatric Council of the American Society of the Echocardiography. Indications and guidelines for performance of transesophageal echocardiography in the patient with pediatric acquired or congenital heart disease: report from the task force of the Pediatric Council of the American Society of Echocardiography. J Am Soc Echocardiogr 2005;18:91-8.

48. Baker GH, Shirali G, Ringewald JM. Usefulness of live threedimensional transesophageal echocardiography in a congenital heart disease center. Am J Cardiol. 2009;103:1025-8.

49. Rawlins DB, Austin C, Simpson JM. Live three-dimensional paediatric intraoperative epicardial echocardiography as a guide to surgical repair of atrioventricular valves. Cardiol Young. 2006;16:34-9.

50. Bleich S, Nanda NC, Hage FG. The incremental value of threedimensional transthoracic echocardiography in adult congenital heart disease. Echocardiography. 2013;30:483-94.

51. Friedberg MK, Su X, Tworetzky W, et al. Validation of 3D echocardiographic assessment of left ventricular volumes, mass, and ejection fraction in neonates and infants with congenital heart disease: a comparison study with cardiac MRI. Circ Cardiovasc Imaging. 2010;3:735-42.

52. Niemann PS, Pinho L, Balbach T, et al. Anatomically oriented right ventricular volume measurements with dynamic threedimensional echocardiography validated by 3-Tesla magnetic resonance imaging. J Am Coll Cardiol. 2007;50:1668-76.

53. • Zamorano JL, Badano LP, Bruce $\mathrm{C}$ et al. EAE/ASE recommendations for the use of echocardiography in new transcatheter interventions for valvular heart disease. Eur Heart J 2011; 32:2189-214. Reference guideline for echocardiographers participating in any or all stages of new transcatheter treatments for patients with valvular heart disease.

54. Messika-Zeitoun D, Serfaty JM, Brochet E, et al. Multimodal assessment of the aortic annulus diameter: implications for transcatheter aortic valve implantation. J Am Coll Cardiol. 2010;55: 186-94.

55. Spencer KT, Krauss D, Thurn J, et al. Transnasal transesophageal echocardiography. J Am Soc Echocardiogr. 1997;10:728-37.

56. Feldman T, Kar S, Rinaldi M, et al. EVEREST Investigators. Percutaneous mitral repair with the MitraClip system: safety and midterm durability in the initial EVEREST (Endovascular Valve Edge-to-Edge REpair Study) cohort. J Am Coll Cardiol. 2009; 54:686-94.

57. Hoffmann R, Kaestner W, Altiok E. Closure of a paravalvular leak with real-time three-dimensional transesophageal echocardiography for accurate sizing and guiding. J Invasive Cardiol. 2013;25:E210-1.

58. Maltais S, Costello WT, Billings FT, et al. Episodic monoplane transesophageal echocardiography impacts postoperative management of the cardiac surgery patient. J Cardiothorac Vasc Anesth. 2013;27:665-9.

59. Cavarocchi NC, Pitcher HT, Yang Q, et al. Weaning of extracorporeal membrane oxygenation using continuous hemodynamic transesophageal echocardiography. J Thorac Cardiovasc Surg. 2013;146:1474-9.

60. Chong A, MacLaren G, Chen R, et al. Perioperative applications of deformation (myocardial strain) imaging with speckle-tracking echocardiography. J Cardiothorac Vasc Anesth. 2014;28:128-40.

61. Geyer H, Caracciolo G, Abe H, et al. Assessment of myocardial mechanics using speckle tracking echocardiography: fundamentals and clinical applications. J Am Soc Echocardiogr. 2010;23: 351-69.

62. Mor-Avi V, Lang RM, Badano LP, et al. Current and evolving echocardiographic techniques for the quantitative evaluation of cardiac mechanics: ASE/EAE consensus statement on methodology and indications endorsed by the Japanese Society of Echocardiography. J Am Soc Echocardiogr. 2011;24:277-313.

63. Kukucka M, Nasseri B, Tscherkaschin A, et al. The feasibility of speckle tracking for intraoperative assessment of regional myocardial function by transesophageal echocardiography. J Cardiothorac Vasc Anesth. 2009;23:462-7.

64. Marcucci CE, Samad Z, Rivera J, et al. A comparative evaluation of transesophageal and transthoracic echocardiography for measurement of left ventricular systolic strain using speckle tracking. J Cardiothorac Vasc Anesth. 2012;26:17-25.

65. Tousignant C, Desmet M, Bowry R, et al. Speckle tracking for the intraoperative assessment of right ventricular function: a feasibility study. J Cardiothorac Vasc Anesth. 2010;24:275-9.

66. • Hahn R, Abraham T, Adams M et al. Guidelines for performing a comprehensive transesophageal echocardiographic examination: recommendations from the American Society of Echocardiography and the Society of Cardiovascular Anesthesiologists. J Am Soc Echocardiogr 2013;26:921-64. Updated guidelines on how to perform a comprehensive intraoperative TEE examination. 
67. - Bose RR, Matyal R, Warraich HJ et al. Utility of a transesophageal echocardiographic simulator as a teaching tool. J Cardiothorac Vasc Anesth 2011;25:212-15. An early paper demonstrating the validity of simulation for teaching TEE.

68. Graber MA, Wyatt C, Kasparek L, et al. Does simulator training for medical students change patient opinions and attitudes toward medical student procedures in the emergency department? Acad Emerg Med. 2005;12:635-9.

69. McGaghie WC, Issenberg SB, Cohen ER, et al. Does simulation based medical education with deliberate practice yield better results than traditional clinical education? A meta-analytic comparative review of the evidence. Acad Med. 2011;86:706-11.

70. - Shakil O, Mahmood F, Matyal R. Simulation in echocardiography: an ever-expanding frontier. J Cardiothorac Vasc Anesth 2012;26:476-85. A review of the simulators available for training in echocardiography.

71. http://pie.med.utoronto.ca/TEE. Accessed 24 July 2014.

72. Weidenbach M, Wild F, Scheer K, et al. Computer-based training in two-dimensional echocardiography using an echocardiography simulator. J Am Soc Echocardiogr. 2005;18:362-6.

73. Matyal R, Bose R, Warraich $\mathrm{H}$, et al. Transthoracic echocardiographic simulator: normal and the abnormal. J Cardiothorac Vasc Anesth. 2011;25:177-81.

74. Shakil O, Mahmood B, Matyal R, et al. Simulation training in echocardiography: the evolution of metrics. J Cardiothorac Vasc Anesth. 2013;27:1034-40.
75. Beer-Gabel M, Delmotte S, Muntlak L. Computer assisted training in endoscopy (C.A.T.E.): from a simulator to a learning station. Endoscopy. 1992;24(Suppl 2):534-8.

76. Stefanidis D, Scerbo MW, Montero PN, et al. Simulator training to automaticity leads to improved skill transfer compared with traditional proficiency-based training: a randomized controlled trial. Ann Surg. 2012;255:30-7.

77. Sainathan S, Andaz S. A systematic review of transesophageal echocardiography-induced esophageal perforation. Echocardiography. 2013;30:977-83.

78. Hilberath JN, Oakes DA, Shernan SK, et al. Safety of transesophageal echocardiography. J Am Soc Echocardiogr. 2010;23:1115-27.

79. Svider PF, Pashkova AA, Vidal GP, et al. Esophageal perforation and rupture: a comprehensive medicolegal examination of 59 jury verdicts and settlements. J Gastrointest Surg. 2013;17:1732-8.

80. Min JK, Spencer KT, Furlong KT, DeCara JM, Sugeng L, Ward $\mathrm{RP}$, et al. Clinical features of complications from transesophageal echocardiography: a single-center case series of 10,000 consecutive examinations. J Am Soc Echocardiogr. 2005;18:925-9.

81. Na S, Kim CS, Kim JY, et al. Rigid laryngoscope-assisted insertion of transesophageal echocardiography probe reduces oropharyngeal mucosal injury in anesthetized patients. Anesthesiology. 2009;110:38-40.

82. Marijon E, Ou P, Celermajer DS, et al. Prevalence of rheumatic heart disease detected by echocardiographic screening. N Engl J Med. 2007;357:470-6. 